\title{
Augmentation of Thermal Performance of Plate Fin Heat Sink
}

\author{
Anilkumar Sathe, Sudarshan Sanap
}

\begin{abstract}
Heat sinks are popularly used in various industrial applications to cool electrical, electronics and automobile components. They are useful in removing the heat from the surfaces at elevated temperatures. The life of such devices depends on their operating temperature. Heat sinks are important parts of thermal management systems of most of these devices eg: Diods, thyristers, high power semiconductor devices such as integrated circuits of inverters, audio amplifier, microprocessors, microcontrollers etc.

In many situations where heat transfer is by free convection where convective heat transfer coefficient is low, fins are the best solution because of their less cost and trouble free operation. The weight and size of equipment are the most important parameters of design. Present day demand, the use of compact systems in every application which leads to higher packing density. The failure rate of electronic equipments increase exponentially with the temperature. Also the high thermal stresses in the solder joints of electronic components mounted on circuit boards resulting from temperature variation are major causes of failure. Therefore thermal control has become an important factor in the design and operation of electronic equipment.

The most preferred method for cooling these systems is passive cooling because it is cost effective and reliable. This leads to focus on development of effective fin heat sink. To make heat sink effective, geometry and orientation of the heat sink as well as heat transfer augmentation techniques plays important role.

This paper highlights the use of heat sinks in electronic cooling applications and review of related literature of improving the heat transfer performance of plate fin heat sinks by surface modifications, interrupting the boundary layer and changing the orientation.
\end{abstract}

Keywords: Heat sink, natural convection, rectangular fins, orientation

\section{INTRODUCTION}

Electronic equipment has made its way into practically every aspect of modern life, from toys and appliances to high power computers. The reliability of the electronic systems is a major factor in the overall working of the system. Electronic components perform their function by passage of electric current and due to the heat generation they become potential sites for excessive heating. Although this "increased power - decreased size" scenario has been prevalent for many decades, the industry's ability to make smaller electronic components mandates reduced size cooling components.

Revised Manuscript Received on July 22, 2019

Anilkumar Sathe, Research Scholar, MIT School of Engineering, Pune, India

Sudarshan Sanap, Research Guide, MIT School of Engineering, Pune,
Market demands have shifted attention to decreasing the volume and increasing thermal efficiency. Total power level is not the only problem. Heat density or Watts per square centimeter, at the electronics devices is a growing problem, too. High heat flux densities combined with high overall power levels are the main problems for thermal management. These thermal challenges can cause equipment failure even if the heat sink surface area and airflow are sized correctly. Unless properly designed and controlled, high rates of heat generation result in high operating temperatures for electronic equipments reducing its safety and reliability.

Air-cooling is the traditional method of cooling electronics. As a heat transfer agent, air is a poor media with the following limitations:

a. Low density and low specific heat, resulting in low heat carrying capacity

b. Low thermal conductivity

However, due to its availability, low cost, ease of maintenance, air-cooling is still the preferred choice.

Improved heat transfer is achieved in either of two ways:

a. Increase the heat transfer coefficient

b. Add more exposed surface

The heat transfer coefficient is a measurement of how effectively heat is removed from a surface. It is expressed in terms of watts dissipated per square meter per degree Celsius of temperature of rise above the cooling medium. Newton's law of cooling states that the rate of heat transfer by convection mode of heat transfer is directly proportional to area available for the heat transfer and temperature difference between surface and surrounding.

$$
\begin{gathered}
Q \propto A\left(T_{s}-T_{\infty}\right) \\
Q=h A\left(T_{s}-T_{\infty}\right)
\end{gathered}
$$

A designer is not having control over the temperature of the surface because it is decided by application. There is no control over surrounding temperature because in most of the cases, surrounding is ambient air. Designer can control only " $A$ ", the number of square centimeters of surface area of the fins, and " $h$ ", the effectiveness of the heat removal from each square centimeter of surface. Both of these parameters are bounded by the limits of cost and marketability. 


\section{Augmentation of Thermal Performance of Plate Fin Heat Sink}

The heat transfer coefficient " $h$ " can be increased by a number of methods:

\section{ELECTRONIC COOLING}

In an electronic system, the electronic components are the sites of heat generation, thus they normally form the hot spots. For controlling temperature of the electronic components effective thermal management is required. The aim of the thermal management is to properly control the operating temperature of the components by safe, efficient and economic removal of the heat. The essence of thermal design is the removal of this internally generated heat by providing an effective path of heat flow from the components to the surrounding medium. Several cooling techniques are commonly used in electronic equipment such as conduction cooling, natural and forced convection, radiation cooling, liquid cooling, immersion cooling and heat pipes. One of the important techniques used is cooling of the electronic equipment is with the help of heat sinks.

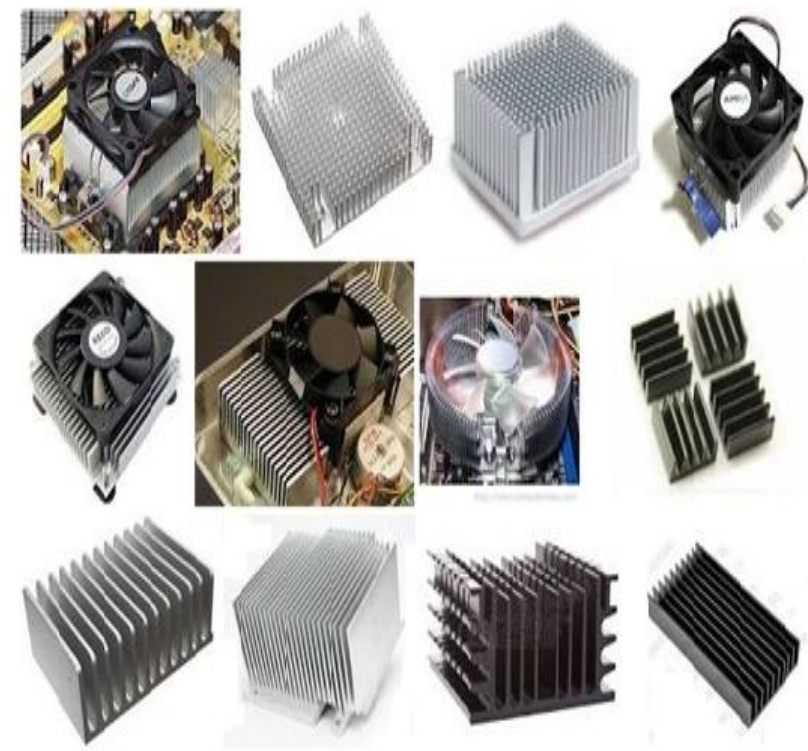

Fig. 1 Heat Sinks

\section{USE OF HEAT SINKS}

Heat sink is an electronic component or a device of an electronic circuit which disperses heat from other components (mainly from the power transistors) of a circuit into the surrounding medium and cools them for improving their performance, reliability and also avoids the premature failure of the components. For the cooling purpose, it incorporates a fan or cooling device.

The heat sinks are useful in various ways as under

i. A heat sink is a passive heat exchanger, and it is designed to have large surface area in contact with the surrounding (cooling) medium like air. The components or electronic parts or devices which are insufficient to moderate their temperature, require heat sinks for cooling. Heat generated by every element or component of electronic circuit must be dissipated for improving its reliability and preventing the premature failure of the component.

ii. It maintains thermal stability in limits for every electrical and electronic component of any circuit or electronics parts of any system. The performance of the heat sink depends on the factors like the choice of a material, protrusion design, surface treatment and air velocity.

iii. The central processing units and graphic processors of a computer are also cooled by using the heat sinks. Heat sinks are also called as heat spreaders, which are frequently used as covers on a computer's memory to dissipate its heat.

iv. If heat sinks are not provided for electronic circuits, then there will be a chance of failure of components such as transistors, voltage regulators, ICs, LEDs and power transistors. Even while soldering an electronic circuit, it is recommended to use heat sink to avoid over heating of the elements.

v. Heat sinks not only provide heat dissipation, but also used for thermal energy management done by dissipating heat when heat is more. In case of low temperatures, heat sinks are intended to provide heat by releasing thermal energy for proper operation of the circuit. Though the heat transfer rate is improved by heat sink or finned system on electronics components lower value of convective heat transfer coefficient is the major concern. Because of low cost and reliability in most of the applications passive cooling is preferred.

As all we know that when the fluid flow over the surface, the boundary layer gets formed along the surface. On the surface of the fin, boundary layer gets formed from leading edge to trailing and it grows along the surface. It will act as resistance for convection heat transfer thus lowers the heat transfer rate. In lower velocities of free convection, the thickness of boundary layer is large as compared to forced convection.

So many of the researchers worked on improving the convective heat transfer coefficient by surface modifications, interrupting the boundary layer and changing the orientation.

\section{LITERATURE SURVEY}

Convection heat transfer rate from fin arrays has been investigated for several geometries in literature both in natural and forced convection mode. The numerical and experimental investigations were performed in order to find the optimum geometric parameters for achieving maximum heat transfer rates from the finned surfaces of heat sink. Most of the recent work by various researchers is summarized and discussed below. 
Low thermal conductivity of air is the main reason for less value of its heat transfer coefficient. Therefore thermal engineers are often interested to find a suitable heat sink that can remove the heat generated efficiently when air cooling is used. Most simple way to carry out this is to more fins on the surface. But initially by adding more number of fins, the thermal performance is enhanced and later it starts deteriorating because of higher frictional resistance and mal-distribution of the contacting fluid. This was well explained by Simon [1]. He studied the effect of number of fins on an Aluminium heat sink of 50 x $50 \mathrm{~mm}$ size, $25 \mathrm{~mm}$ length of fin and $0.5 \mathrm{~mm}$ thickness of fin as shown in figure 2. He observed that thermal resistance decreases initially with number of fins. Adding fins more than 22 is not acceptable as it increases thermal resistance. Moreover he observed that by adding more fins on the surface decreases the flow rate. This counter acts the benefit obtained by adding the surface area. Also adding number of fins increases weight and cost of the heat sink. The conclusion of the Simon's work is by adding more fins in not the only option to improve thermal performance of heat sink.

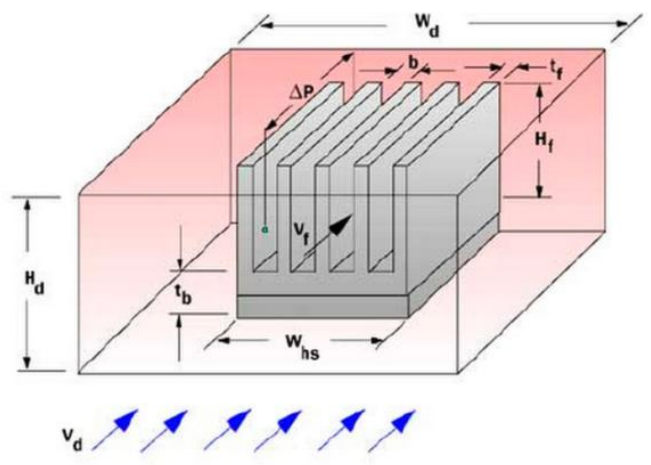

Fig. 2 Heat sink with geometry with number of fins

Hung Yi Li and Chao [2] measured performance of plate fin heat sink with cross flow cooling. Experimental results indicate that increase in Reynolds number decreases the thermal resistance. They observed that pressure drop increases with increase in Reynolds number, fin width and fin height. Study also reveals that increase in fin width degrades the thermal performance.

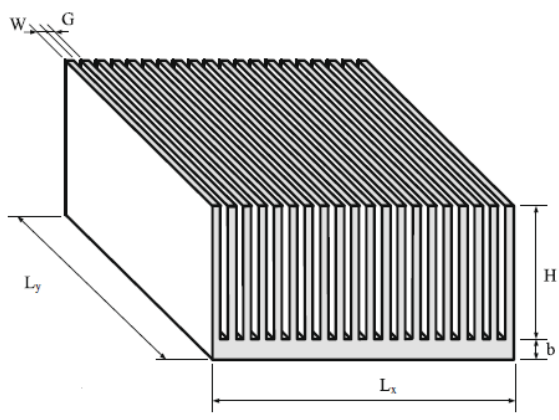

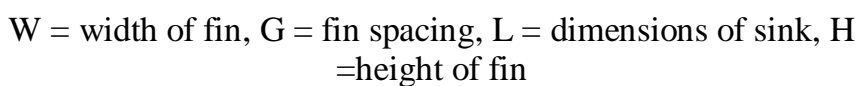

Fig. 3 Geometry of heat sink
Abbas Jubear and Hamadani [3] investigated the effect of fin height on thermal performance of plate fin array in natural convection. Study reveals that the heat transfer performance increases with fin height for constant fin spacing. They established correlation between Nusselt number and fin height by keeping all other geometrical parameters constant as $N u=7 R a^{0.3} H^{0.7}$ for fin height varied between 10 to 45 $\mathrm{mm}$.

Newton's law of cooling suggests that the heat transfer rate can be increased by increasing convective heat transfer coefficient. Many researchers tried to increase convective heat transfer coefficient through mechanisms of boundary layer restarting or creating vortices in the path of fluid. Yang et al. [4] examined effect of some highly interrupted fin surfaces using slitted fins or louvers as depicted in figure 4. They observed that because of renewal of boundary layer enhances the thermal performance. However they found that it works for fin spacing greater than $1.6 \mathrm{~mm}$. For lower value of fin spacing like $0.8 \mathrm{~mm}$, significant pressure drop takes place especially for low value of Reynolds number.
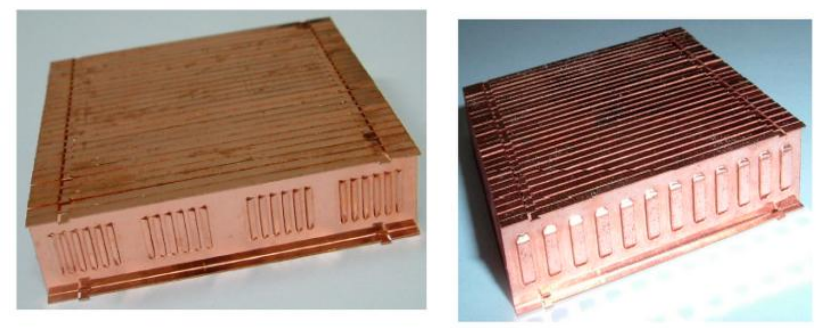

Fig. 4 Heat sink with Louvers and slits

In addition to louvers and slits some researchers tried the use of swirl flow geometries or vortex generators on the surface of fin. Yang et al. [5] studied the use of delta wing, triangular winglets, semicircular winglets and dimples with variable spacing. Tested geometries are given in figure 5 .

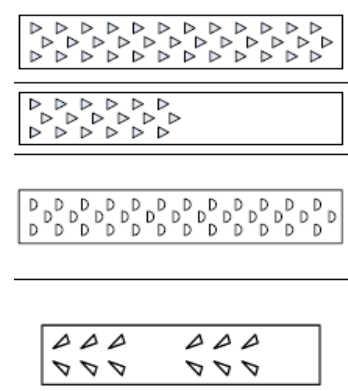

Fig. 5 Triangular, semicircular and triangular winglets on fin surface

The test results show that semicircular winglets give higher pressure drop as compared to other geometries. The pressure drop also depends on density of geometry on the fin surface. The experimentation also reveals that the resultant pressure drop is much lower than the interrupted surfaces.

Less effectiveness of interruptedsurface and vortex generators at low value of Reynolds number motivated few other researchers to think about novel cannelure fin structure. 


\section{Augmentation of Thermal Performance of Plate Fin Heat Sink}

Wang et al. [6] developed the cannelure fin structure with fin spacing is $0.8 \mathrm{~mm}$ for test heat sinks. The idea is to decrease pressure drop at low velocity. They showed approximately 25 $\%$ enhancement in heat transfer enhancement however corresponding pressure drop is reduced by $20 \%$.
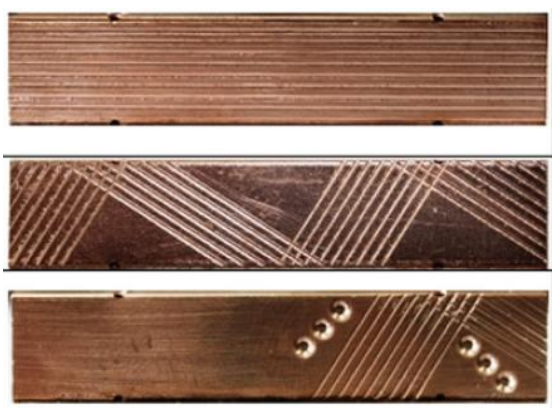

Fig. 6Novel cannelure fin structure and its combination with dimples

Dhanawade and et al. [7] examined the heat transfer enhancement over horizontal flat surface with rectangular fin arrays having square and circular perforation in forced convection. Study finds that the square perforations are superior to circular perforations.

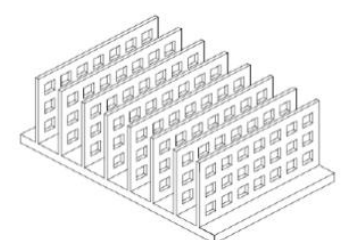

Fig. 7 Circular and square perforations

Shyy-Woei Chang et al. [8] numerically examined the steady-state three-dimensional natural convective flow and heat transfer for a set of vertical fin arrays with and without dimples. The finite Navier-Stokes and energy equations using semi-implicit method for pressure-linked equation (SIMPLE) with the converged solutions from the iterative steps to acquire the velocity field, temperature field, and Nusselt number $(\mathrm{Nu})$. The free convective flow and heat transfer for different vertical fin arrays are analyzed at four different sets of Rayleigh numbers (Ra) with the fixed Prandtl number. For each $\mathrm{Ra}$ tested, under the condition of same fin base area and fin-array volume, they found that heat transfer performances of dimpled fin array is better than smooth fin array. As $\mathrm{Ra}$ increases, the mean $\mathrm{Nu}$ over each fin surface increases, especially for the dimpled fin arrays. Relative to the smooth thirteen fin array, the maximum increase of mean $\mathrm{Nu}$ is $68 \%$ for the dimpled nine-fin array

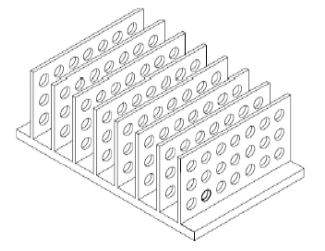
volume method is adopted to solve the

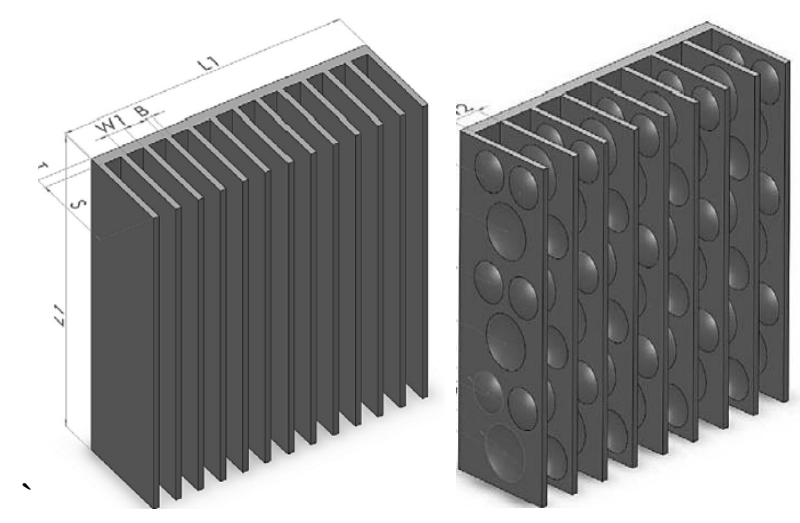

Fig. 8 Plane plate fins and dimpled plate fins

Younghwan Joo and Sung Jin Kim [9] compared experimentally, performance of optimized pin fin array and optimized rectangular plate fin array. The comparison was made under the same base-plate dimensions and fin height conditions. A new correlation of the heat transfer coefficient is proposed and validated experimentally to optimize pin-fin heat sinks, while a correlation of the heat transfer coefficient for plate-fin heat sinks is adopted from previous studies. They found that the optimized plate-fin heat sinks dissipate a larger amount of total heat than do the optimized pin-fin heat sinks in most practical applications.

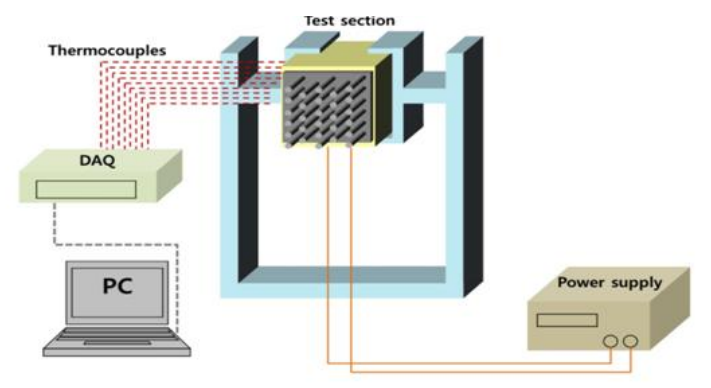

Fig. 9 Experimental setup

Umesh V. Awasarmol and Ashok T. Pise [10] carried out experimental study is to quantify and compare the natural convection heat transfer enhancement of perforated fin array with different perforation diameter $(4-12 \mathrm{~mm})$ and at different angles of inclination $\left(0-90^{\circ}\right)$. The variables for this natural convection cooling with the help of finned surfaces are orientation and geometry. In this study, the steady state heat transfer from the solid fin and perforated fin arrays are measured. The increase in the heat transfer coefficient was achieved with perforated fins of $12 \mathrm{~mm}$ perforation diameter at the angle of orientation $45^{\circ}$, which shows about $32 \%$ enhanced heat transfer coefficient as opposed to the solid fin array with nearly $30 \%$ saving in material by mass 


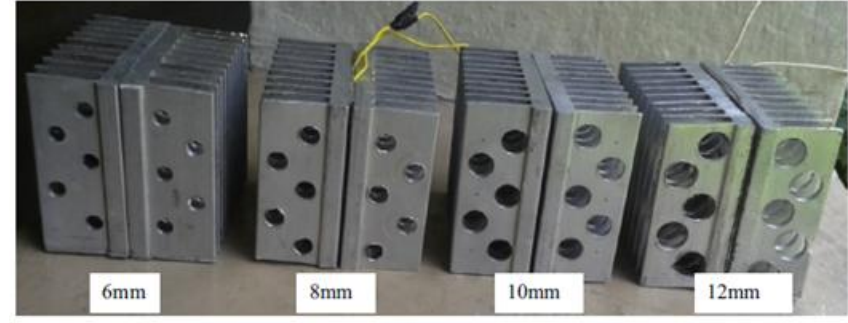

Fig. 10 Round perforations

Roody Charles and Chi-Chuan Wang [11] - In this study, a comparative study of heat sink having various fin assembly under natural convection is investigated. Researchers used fin patterns as rectangular, trapezoidal and an inverted trapezoidal configuration. Tests were performed in a well controlled environmental chamber having a heat load ranging from 3 to $20 \mathrm{~W}$. From the test results, the heat transfer coefficient of the conventional rectangular fins is higher than that of the trapezoidal fins while the heat transfer coefficient of the inverted trapezoidal fins is higher than the trapezoidal one by approximately $25 \%$, and it exceeds that of convectional rectangular fin by about $10 \%$. The heat transfer improvements of the inverted trapezoidal fin are mainly associated with a larger temperature difference and inducing more air flow into the heat $\operatorname{sink}$

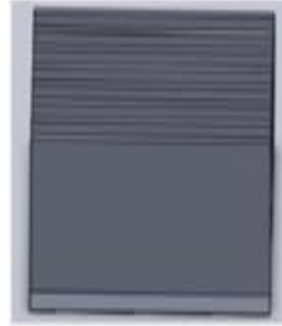

(a) Rectangular fin

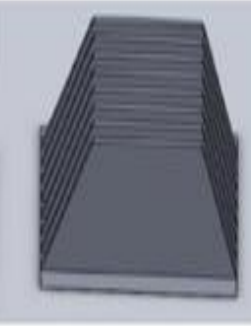

(b) Trapezoidal fin

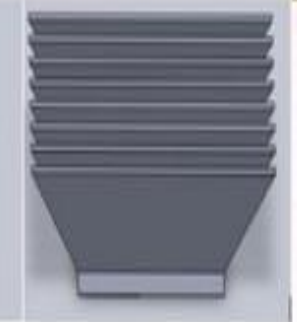

(c) Inverted trapezoidal fin
Fig. 11 Different Geometry of plate fins

Murtadha Ahmed and Abdul Jabbar N. Khalifa [12] carried out experiments on natural convection heat transfer from square pin fin heat sinks subject to the influence of its geometry and heat flux. A total of 50 fins were bolted into the upper surface of the base plate. The area of the base plate is $250 \mathrm{~mm}$ by $250 \mathrm{~mm}$. Over the tested range of Rayleigh number, it was found that the solid pin fin heat sink performance for upward orientations depended on the Rayleigh number and generally shows higher heat transfer coefficients than those of the perforated pin fin. For all tested hollow/perforated pin fin heat sinks, however, the heat transfer performance for heat sinks with hollow/perforated pin fins was better than that of solid pins. The temperature difference between the base plate and surrounding air of these heat sinks was less than that of solid pin one.

\section{(a) Pif fins, (b) Base plate, (c)Heater, (d)Firebrick, (e)Glass wall blanket, (f)Wooden box}

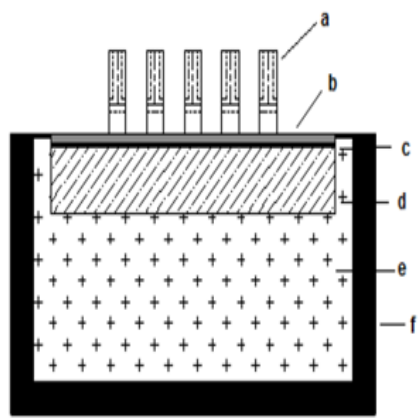

Fig. 12 Heat sink assembly

Kanargi etal.[13] carried out numerical and experimental investigation of thermal performance of cross connected alternative converging and diverging channel heat sink and compared the results with the plate fins. They shown that arrangement induces secondary flow in the fluid through the cross connections repeatedly disturbs the boundary layers. However vortices generated as a result of secondary flows were observed to prevent heat transfer performance from being further improved and results into excessive increase in pressure drop.

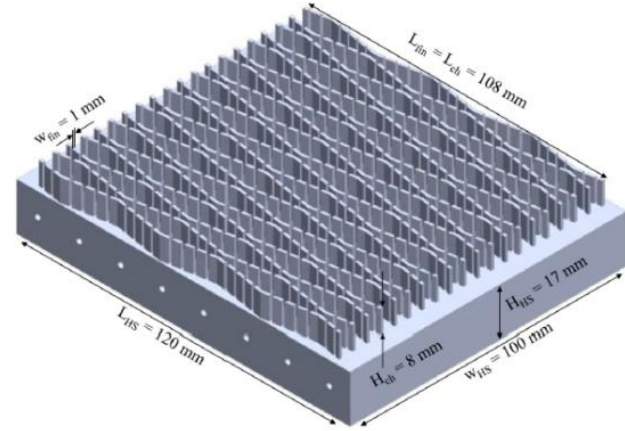

Fig. 13 Cross connected alternating converging-diverging heat sink

S. G. Taji et al. [14] experimentally investigated the effect of fin spacing on thermal performance heat sink in horizontal configuration. Dimensionless fin spacing to height $(\mathrm{S} / \mathrm{H})$ ratio is varied from 0.05 to 0.3 and length to height ratio $(\mathrm{L} / \mathrm{H})=$ 5 is kept constant. The heater input to the fin array assembly is varied from 25 to $100 \mathrm{~W}$. The single chimney flow pattern is observed from 8 to $12 \mathrm{~mm}$ fin spacing. The end flow is choked below $6 \mathrm{~mm}$ fin spacing.

Guei-Jang Huang et al. [15] studied the effect of introducing perforations through the fin base to improve ventilation with cold air from below the fin base. The fin-base perforations, especially locating in the inner region, improve ventilation and heat transfer performance of large fin arrays significantly. For long fin arrays with a length of $380 \mathrm{~mm}$, the overall heat transfer coefficients are enhanced by a factor of 1.49 to 2.77 , 


\section{Augmentation of Thermal Performance of Plate Fin Heat Sink}

depending on the total perforation length and the perforation pattern. But the benefit of base-plate perforations is not as remarkable for short fin arrays as for long ones. In addition they found that the overall convective heat transfer coefficients of the perforated fin arrays increase with increasing total perforation length.

Xiaolig $\mathrm{Yu}$ et al. [16] suggested a new type plate fin heat sink which is a combination of pin fins staggered between plate fins. Numerical and experimental investigation reveals that plate pin fin arrangement is superior to plate fin arrangement as thermal resistance of former is 30 $\%$ lower than that of later counterpart. The pin fin columns acts as air flow disturbers improving thermal performance at the cost of additional pressure drop. For the same pumping factor, the performance of plate pin fin arrangement is 20 $\%$ higher than plate fin arrangement
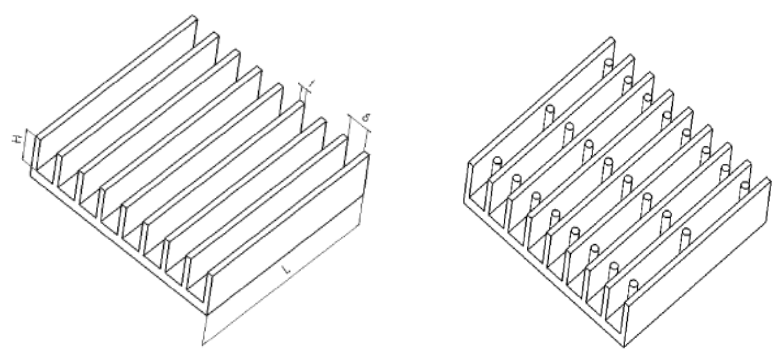

Fig. 14 Plate fin and plate pin fin arrangements

Anant Joshi and D. G. Kumbhar [17] carried out experimentation which predicts the flow of air over the fin array and the heat transfer due to natural convection. Fin array is modeled using Pro/E and material used is aluminum. Nine rectangular fins are used and placed over a heat source. They considered different heat inputs and experiments were performed for fin array without notches and with notches. Notches with square cross section were used for experimentation. Variation in notch cross section was provided by reducing the notch area by $10 \%, 20 \%$ and $30 \%$ respectively. Results show that the value of heat transfer coefficient increases with the increase in the notch area. As area removed from the fin is compensated at the air entry ends of the fin, it provides a chance to get greater amount of fresh cold air to come in contact with hot fin surface. As the air moves inwards it gets heated and temperature difference between the fin and entering air decreases.

H. Deshpande and S. G. Taji [18] investigated the effect of fin spacing and perforations on thermal performance of horizontal plate fin heat sink. Experimental results shows that optimum fin spacing is around $10 \mathrm{~mm}$ and perforations created at the center of fin surface are useful to augment convective heat transfer coefficient by $15 \%$. The correlation was formulated based on selected range of heat input and geometrical parameters

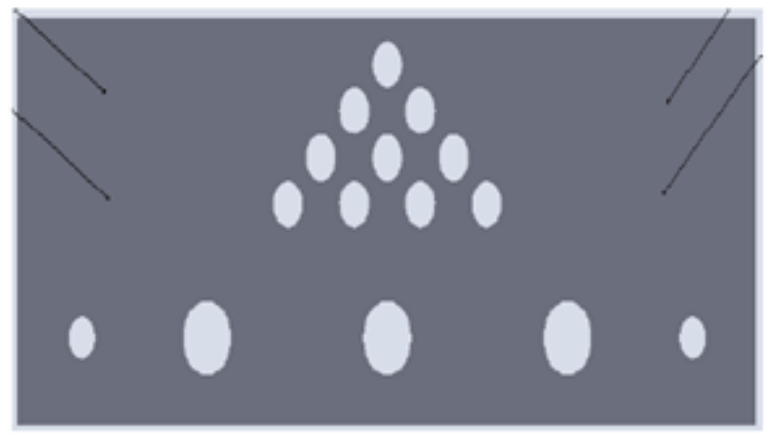

Fig. 15 Perforations at center of fin surface

Ilker Tari and Mehdi Mehrtash [19] studied natural convection from plate-fin heat sinks in both horizontal and slightly inclined from horizontal orientations. A set of correlations was obtained for both upward and downward facing horizontal cases. The correlations were tested for validity by using large sets of experimental data from literature. By varying the direction of gravitational acceleration for several upward and downward inclinations, the phenomenon is investigated for the purpose of determining the flow structures forming in and around the heat sinks. A complete set of Nusselt number correlations covering all possible inclination angles was suggested.

Kim et al. [20] investigated the effect of various fin numbers, fin heights, and base temperatures on natural convection from horizontal cylinders with longitudinal plate fins experimentally. Experimental results were used to develop a correlation under the parameters used for test. Results were further used to develop a contour map showing relationship between thermal resistance, fin number and fin thickness.

Naserian et al. [21] studied the shape and the number of pieces and the gap between the pieces in different cases of V-type fin configuration in laminar natural convection condition, both experimentally and numerically. They concluded that by increasing the fin number and the fin spacing, the ratio of natural convection heat transfer coefficient of various fin configurations to the corresponding vertical plate is increased. The results show that the maximum natural convection coefficient and also the maximum natural convection heat transfer rate, takes place for the case in which the fin has the least effect on the air flow in the vicinity of base plate and also the thickness of thermal boundary layer on the fin is fine. In addition, study was carried out to understand the effect of row spacing.

Shivdas S. Kharche and Hemant S. Farkade [22] experimentally and theoretically investigated natural convection heat transfer from vertical rectangular fin arrays with and without notch at the center. Moreover notches of different geometrical shapes have also been analyzed for the purpose of comparison and optimization. From the experimental study it is found that the heat transfer rate in notched fins is more than the unnotched fins. The average heat transfer coefficient for without notched fin is 8.3887 $\mathrm{W} / \mathrm{m}^{2} \mathrm{~K}$ and for $20 \%$ notched fins it is $9.8139 \mathrm{~W} / \mathrm{m}^{2} \mathrm{~K}$.

Xiaohui Zhang and Dawei Liu [23] investigated the optimal spacing between isothermal laminar natural convection plates cooled by air for maximal heat transfer analytically and numerically. It is found that the optimal plate's allocation spacing is different from the

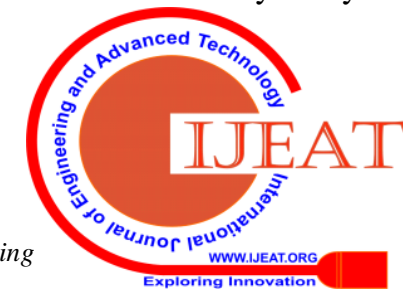


conventional way, where the boundary layers of the plates merge early; it is the distinguishing feature of the outlet velocity that causes an enhancement of heat transfer.

E. A. M. Elshafei [24] were performed experiments on natural convection heat transfer from circular pin fin heat sinks subject to the influence of its geometry, heat flux and orientation. The geometric dependence of heat dissipation from heat sinks of widely spaced solid and perforated circular pin fins with staggered combination, fitted into a heated base of fixed area is discussed. Over the tested range of Rayleigh number, $3.8 \times 10^{6} \leq \mathrm{Ra} \leq 1.65 \times 10^{7}$, it was found that the solid pin fin heat sink performance for upward and sideward orientations shows a competitive nature, depending on Rayleigh number and generally shows higher heat transfer coefficients than those of the perforated/ hollow pin fin ones in both arrangement. For all tested hollow/perforated pin fin heat sinks, however, the performance for sideward facing orientation was better than that for upward facing orientation. Ren-Tsung Huang et al. [25] carried out experiments on natural convection heat transfer from square pin fin heat sinks subject to the influence of orientation. A flat plate and seven square pin fin heat sinks with various arrangements are tested under a controlled environment. Test results indicate that the downward facing orientation yields the lowest heat transfer coefficient. However, the heat transfer coefficients for upward and sideward facing orientations are of comparable magnitude. Depending on the fin structure, the performance of these two orientationsshows a competitive nature. It is found that the sideward arrangement outperforms the upward one for small finning factors below 2.7, beyond which the situation is reversed. In addition, with the gradual increase in the finning factor, the performance of sideward arrangement approaches that of downward arrangement.

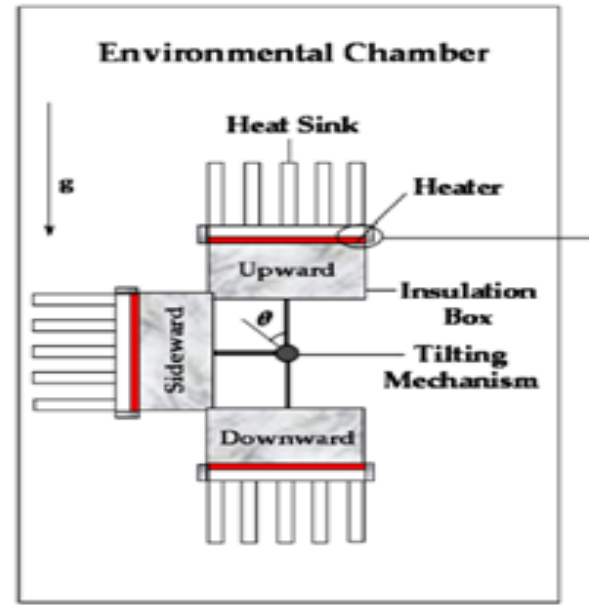

Fig. 16 Arrangement of orientations

Chen and Wang [26] tested various arrangements of straight fin, step fin and trapezoidal fin as shown in figure 17. The different geometry is based on the heat transfer enhancement through (a) longer perimeter of entrance region and (b) larger effective temperature difference at the rear part of the heat sink. From the test results, it is concluded that that step or trapezoid geometry can provide a higher thermal performance and a lower pressure drop at a specified frontal velocity.

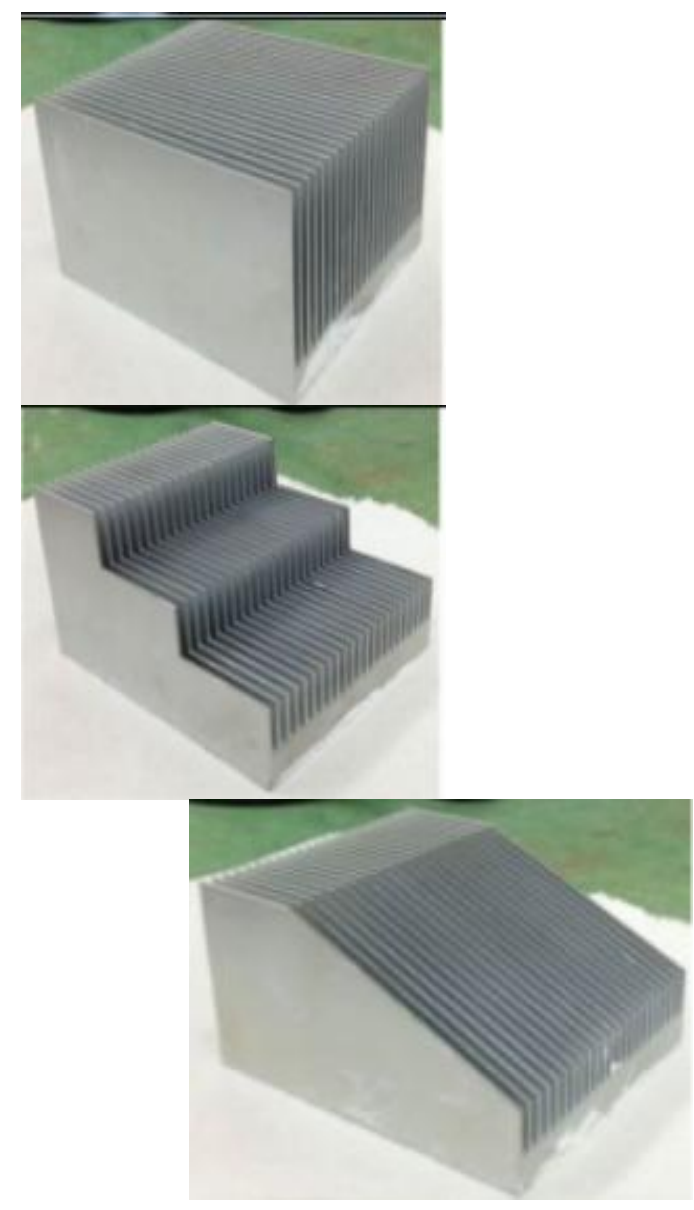

Fig. 17 Different geometries of plate fin

Abdullah H. M. and AlEssa [27] examined heat transfer enhancement from a horizontal rectangular fin embedded with square perforations under natural convection compared to the equivalent solid fin. The study reveals that at certain dimensions of perforations and spacing, the thermal performance is higher than its solid counterpart. The heat transfer enhancement of the perforated fin increases as the fin thickness is increased. . The perforation of fins not only enhances heat dissipation rates but at the same time decreases the expenditure of the fin material.

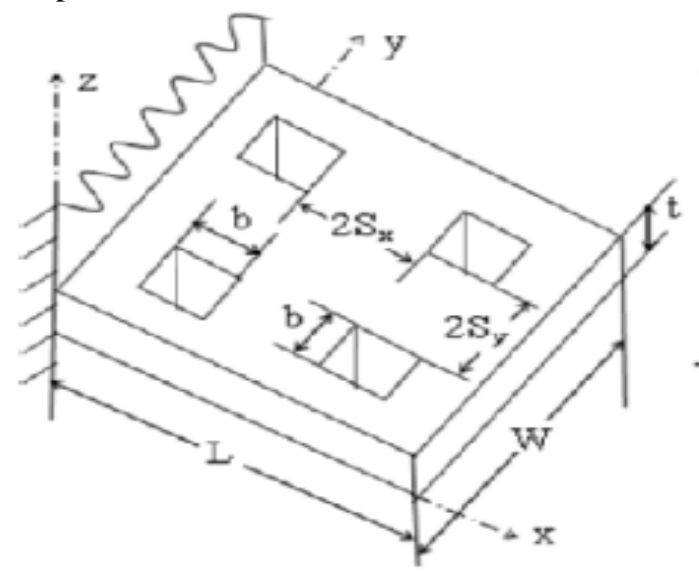

$\mathrm{b}=$ perforation dimension, $\mathrm{S}=$ fin spacing, $\mathrm{W}$ and $\mathrm{L}=$ fin dimensions

Fig. 18 Fin with square perforations 


\section{Augmentation of Thermal Performance of Plate Fin Heat Sink}

\section{CONCLUSION}

1. Many research papers were published regarding various arrangements of heat sinks under natural (free) or forced convection modes.

2. The geometrical parameters of heat sink such as fin thickness, fin length, fin spacing, fin shape and orientation plays important role on the thermo hydraulic performance. Researchers investigated the effect of these geometrical parameters experimentally; some of them predicted results numerically.

3. Most of the researchers have used natural convection because of low value of convective heat transfer coefficient for air.

4. Many of the researcher tried horizontal configuration because of different applications in electronics industry.

5. Some researchers were used surface modifications techniques that introduce the vortices in the air flow to augment the thermal performance. Most of them were concentrated on disturbing the boundary layers by surface modifications or changing the orientation of the heat sink.

6. There lies an opportunity to carry out the more research in augmenting the heat transfer by breaking the boundary layer instead of disturbing.

7. There is more scope to carry out the study on vertical fin configuration which can be used on inverters, LED panels, audio systems etc. Also there is more scope to perform experimental study of the above method and its validation by numerical investigation.

\section{REFERENCES}

1. Simon, R.E., "Estimating the effect of flow bypass on parallel plate fin heat sink performance". Electronic cooling, 1 February 2004. Available online: http://www.electronics-cooling.com

2. Hung Yi Li and Chao, "Measurement of performance of plate-fin heat sinks with cross flow cooling", International Journal of Heat and Mass Transfer, 2009

3. Abbas Jubear and Hamadani, "The effect of fin height on free convection heat transfer from rectangular fin array", International Journal of Recent Scientific Research Vol. 6, Issue 7, pp. 5318-5323, July -2015

4. Yang et al., "On the heat transfer characteristics of heat sinks: Influence of fin spacing at low Reynolds number region", International Journal of Heat and Mass Transfer, 2006

5. Yang et al., "Analysis and optimum for air cooling thermal module using dimple vortex generators", Proceedings of the 14th International Heat Transfer Conference, IHTC14, August 8-13, 2010, Washington, DC, USA

6. Wang et al., "Effect of cannelure fin configuration of compact air cooling heat sink", Applied Thermal Engineering,1640-1647, 31, 2011

7. Dhanawade et al., "Thermal Analysis of Square and Circular Perforated Fin Arrays by Forced Convection", International Journal of Current Engineering and Technology, 2, Feb - 2014

8. Shyy-Woei Chang et al., "Heat transfer enhancement of vertical dimpled fin array in natural convection", International Journal of Heat and Mass Transfer, 2016

9. Younghwan Joo and Sung Jin Kim, "Comparison of thermal performance between plate-fin and pin-fin heat sinks in natura convection", International Journal of Heat and Mass Transfer, $345-356,83,2015$

10. Umesh V. Awasarmol and Ashok T. Pise, "An experimental investigation of natural convection heat transfer enhancement from perforated rectangular fins array at different inclinations", Experimental Thermal and Fluid Science, 145-154, 68, 2015
11. Roody Charles and Chi-Chuan Wang, "A novel heat dissipation fin design applicable for natural convection augmentation", International Communications in Heat and Mass Transfer, 24-29, 59, 2014

12. Murtadha Ahmed and Abdul Jabbar N. Khalifa, "Natural Convection Heat Transfer from a Heat Sink with Fins of Different Configurations", International Journal of Innovation and Applied Studies, 1043-1047, 9(3), 2014

13. Kanargi et al., "A numerical and experimental investigation of heat transfer and fluid flow characteristics of a cross-connected alternating converging-diverging channel heat sink", International Journal of Heat and Mass Transfer, 106, 449-464, 2017

14. S. G. Taji and N. K. Sane, "Experimental investigation of heat transfer and flow pattern from heated horizontal rectangular fin array under natural convection", Heat Mass Transfer, 1005-1015, 50, 2014

15. Guei-Jang Huang et al., "Enhancement of natural convection hea transfer from horizontal rectangular fin arrays with perforations in fin base", International Journal of Thermal Sciences, 164-174, 84, 2014

16. Xiaolig Yu et al., "Development of a plate-pin fin heat sink and its performance comparisons with a plate fin heat sink", Applied Thermal Engineering, 25, 173-182, 2005

17. Anant Joshi and D. G. Kumbhar, "Analysis of Heat Transfer From Horizontal Rectangular (Square Notched) Fin Arrays by Natural Convection", International Journal of Engineering Innovation \& Research, 4(1), 2014

18. H. Deshpande and S.G. Taji, "Experimental Study of Heat Transfer from Horizontal Rectangular Fins with Perforations under Natural Convection", International Journal of Engineering Research \& Technology, 2026-2031, 3(2), 2014

19. Ilker Tari and Mehdi Mehrtash, "Natural convection heat transfer from horizontal and slightly inclined plate-fin heat sinks", Applied Thermal Engineering, 2013

20. Hyun Jung Kim et al., "Experimental study on natural convection heat transfer from horizontal cylinders with longitudinal plate fins", Journal of Mechanical Science and Technology, 593-599, 27(2), 2013

21. Mohammad Mahdi Naserian et al., "Experimental and numerical analysis of natural convection heat transfer coefficient of V-type fin configurations", Journal of Mechanical Science and Technology, 2191-2197, 27 (7), 2013

22. Shivdas S. Kharche and Hemant S. Farkade, "Heat Transfer Analysis through Fin Array by Using Natural Convection", International Journal of Emerging Technology and Advanced Engineering, 595-598, 2(4), 2012

23. Xiaohui Zhang and Dawei Liu, "Optimum geometric arrangement of vertical rectangular fin arrays in natural convection", Energy Conversion and Management, 2449-2456, 51, 2010

24. E.A.M. Elshafei, "Natural convection heat transfer from a heat sink with hollow/perforated circular pin fins", Energy, 2870-2877, 35, 2010

25. Ren-Tsung Huang et al., "Orientation effect on natural convective performance of square pin fin heat sinks", International Journal of Heat and Mass Transfer, 2368-2376, 51, 2008

26. Chen, C.H., Wang, C.C., "A novel trapezoid fin pattern applicable for air-cooled heat sink", Heat Mass Transfer, 51, 1631-1637, 2014

27. Abdullah H. M. and AlEssa, "Augmentation of Fin Natural Convection Heat Dissipation by Square Perforations", Journal of Mechanical Engineering and Automation, 1-5, 2(2),2012 